\title{
"Comparing outcomes of advanced nurse practitioners to ophthalmologists performing posterior YAG capsulotomy, a six-year study of 6308 eyes"
}

\author{
George Moussa (iD ${ }^{1 凶}$, Dimitrios Kalogeropoulos ${ }^{1,2}$, Soon Wai Ch'ng ${ }^{1}$, Jesse Panthagani ${ }^{1}{ }^{1}$, Ziad Abdel-Karim ${ }^{1}$ and Walter Andreatta ${ }^{1,3,4}$
}

(c) The Author(s), under exclusive licence to The Royal College of Ophthalmologists 2022

PURPOSE: To primarily report the baseline characteristics and visual acuity (VA) outcomes of advanced nurse practitioners (ANP) compared to ophthalmologists following YAG posterior capsulotomy (YAGPC). We secondarily looked to characterise the risk factors that lead to a repeated YAGPC.

METHOD: Retrospective consecutive case series of 6,308 eyes attending the Birmingham and Midlands Eye Centre. RESULTS: ANPs performed $33.1 \%$ of YAGPC compared to $66.9 \%$ ophthalmologists. Compared to ophthalmologists, ANPs performed YAGPC in lower proportion of patients with ocular co-morbidities $(p<0.001)$ and had lower proportion of patients requiring further YAGPC compared to ophthalmologists $(p<0.001)$. Median pre, post-operative and LogMAR gain in VA of 0.48 (IQR $0.30-0.78), 0.18$ (IQR $0.10-0.40)$ and $0.30(0.08-0.48)$ LogMAR units, respectively. Multivariate regression showed that ANPs had a significantly lower rate of repeat YAGPC compared to ophthalmologists even when adjusting for age, ethnicity, training grade and ocular co-morbidities. No difference in visual outcomes was found between operator grade and ethnicity on multivariate analysis. CONCLUSION: YAGPC leads to excellent visual outcomes. ANPs can deliver safe and effective YAGPC.

Eye (2023) 37:554-559; https://doi.org/10.1038/s41433-022-01986-8

\section{INTRODUCTION}

Posterior capsule opacification (PCO) is one of the most common post-operative complications of cataract surgery $[1,2]$. PCO is the result of abnormal proliferation, growth and differentiation of residual lens epithelial cells (LECs) that migrate from the equator of the capsule over the posterior capsule leading to obscuration of the visual axis $[2,3]$. Despite advances in surgical techniques, improvement of intraocular lens (IOL) design, and introduction of pharmaceutical agents to inhibit PCO, this condition continues to remain as a common postoperative complication. Taking into consideration the undesirable effects on vision and the costs of treating PCO, a significant amount of research has been done to recognize the main pathogenetic factors, and therapeutic approaches for its prevention and management [4]. More recent reviews have not revealed any substantial differences in PCO scores for different IOL materials but have shown significantly lower PCO rates in IOLs with sharp posterior edges regardless of their material [1]. It has been shown that a 360-degree sharp posterior optic edge pressing against the capsule causes a bend that creates a hindrance to the epithelial cell migration across the lens [5-18]. Neodymium:YAG (Nd:YAG) laser posterior capsulotomy (YAGPC) is the most widely performed definitive treatment for PCO [1]. The rate of eyes requiring NG:YAG laser capsulotomy (YAGPC) following cataract surgery is reported to be $2.4-12.6 \%$ at three years and $7.1-22.6 \%$ at five years [19]. The time lapsed after cataract surgery is an important risk factor in the development of PCO [19].

YAGPC is a common ophthalmic procedure and has a low risk factor profile $[20,21]$. Advanced nurse practitioners (ANP) have successfully been integrated into the National Health Service (NHS) for intravitreal injections with excellent patient satisfaction [22]. While ANPs have had an increasing role as operators in YAGPC, no previous study evaluated the outcomes of ANPs in performing this procedure. Therefore, in this series we evaluated the outcomes of an ANP in conducting this laser intervention, comparing the results to those of Ophthalmologists. In addition, we present the largest series on present day visual outcomes following YAGPC.

\section{METHODS}

We present a single centre, retrospective, continuous case series to analyse all patients that had primary YAG Capsulotomy from January 2014 to July 2020. Every YAGPC was performed with a Litechnica Lpulsa Q-YAG Laser machine. YAGPC was achieved through either a cross or circle pattern, depending on the operator's preference. Repeat YAGPC was performed if the visual axis could not be cleared following initial treatment.

All data was extracted from electronic patient records (EPR, Medisoft Ophthalmology, Medisoft Limited, Leeds, UK). Data collection included ethnicity, age, gender, laterality, number of further YAGPC episodes, operator grade, complications pre-operative, and last recorded post-

\footnotetext{
${ }^{1}$ Birmingham Midlands Eye Centre, Sandwell and West Birmingham Hospitals NHS Trust, Dudley Road, Birmingham B18 7QH, UK. ${ }^{2}$ Department of Ophthalmology, Faculty of

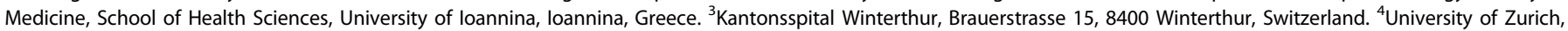
Rämistrasse 71, 8006 Zurich, Switzerland. ${ }^{\circledR}$ email: george.moussa@nhs.net
}

Received: 24 January 2021 Revised: 29 January 2022 Accepted: 10 February 2022

Published online: 28 February 2022 
operative visual acuity (VA). Operator grade was split as five groups: (i) non-training grade ophthalmologists (Staff Grade, Associate Specialist and Specialty Doctors [SAS]), (ii) Advanced Nurse Practitioners (ANP) (iii) Residents (iv) Fellows and (v) Consultants. These groups are presented in the figures and tables for a detailed breakdown of operator grade. Some comparisons and statistical analyses are performed on ANP and ophthalmologists as dichotomised groups.

Preoperative VA was the most recent measurement taken prior to the YAGPC. Post-operative VA was the last recorded vision from two weeks to one year after the YAGPC was performed. The prolonged duration of follow-up in this study was due to the necessity of postposing follow-up appointments of elective cases during the COVID-19 pandemic. Visual loss was defined as a postoperative reduction in VA of $\geq 0.30$ LogMAR units. As visual improvement we considered a VA gain of $\geq 0.30$ LogMAR units at the last follow-up appointment.

To assess the safety profile of YAGPC performed by our ANP compared to Ophthalmologists, we primary looked at visual outcomes, complication rates and repeat YAGPC rate. These were also assessed under a multivariate regression model to provide risk adjusted comparisons.

\section{Advanced nurse practitioner training}

In our unit, to enable enrolment in the YAG training program an ANP must have:

Worked at a nurse specialist level, possessing slit-lamp skills.

Completed a specialist ophthalmic nursing diploma course, independent prescribing course, delegated consent form training and the laser core of knowledge course.

Attended a YAG - laser theory and practical course.

In-depth knowledge of the local laser safety protocols and standard operating procedures (SOP).

The ANP in our unit (author ZA) also underwent a YAG laser machine training on its operation and safety, provided by the Litechnica company engineer. He observed how another ANP based at Cheltenham General Hospital performed YAGPCs, with the aid of a live screen display. He performed YAGPCs for six months in our unit under the supervision of experienced ophthalmologists. He completed 40 supervised cases before being signed off by our laser consultant lead. He has, at the time of writing, six years of experience in performing YAGPC.

\section{Statistical analysis}

Statistical significance was defined as $p<0.05$. Prior to analysis, normality of continuous variables was assessed using the Shapiro-Wilk test and found not to be normally distributed. Hence, data are primarily reported as medians and interquartile ranges (IQRs) throughout. Mann-Whitney $U$ and Kruskal-Wallis Test were used to compare two and more-than two independent groups respectively (age and VA). Wilcoxon signed rank test was used for two-paired VA data. Fisher exact test and Chi-Squared test were used for nominal variables. A multivariate binary logistic regression analysis was performed to calculate odds ratio (OR) and 95\% confidence intervals $(\mathrm{Cl})$ to assess risk factors contributing to repeat YAGPC (dependent variable) and complications following primary YAGPC. A multivariate linear regression model was also performed to investigate visual outcomes. Operator level, presence of ocular co-morbidity, ethnicity subgroups, and age were used as independent variables in all models. Best corrected VA was used and records in Snellen Fraction were converted to LogMAR. Low VA, corresponding to count fingers (CF), hand movements (HM), light perception (LP) and no LP (NLP) were substituted with 2.10, 2.40, 2.70 and 3.00 LogMAR, respectively, in keeping with previous publications from the UK National Ophthalmology Database (NOD) group [23] using an Excel based tool by Moussa et al. [24]. All statistical analysis was performed using IBM SPSS Statistics for Windows, Version 27.0 (IBM Corp, Armonk NY).

\section{RESULTS}

\section{Baseline demographics and characteristics}

We report outcomes in 6308 eyes undergoing primary YAGPC. Within our cohort, 2688 (42.6\%) were males and 1692 (26.8\%) were noted to have reduced visual potential due to ocular comorbidities. YAGPC was performed bilaterally in 899 (14.3\%).

One hundred and eleven ophthalmologist operators contributed to our data, compared to one ANP. We found that $33.1 \%$ of all primary YAGPC were performed by our ANP. A summary of the baseline characteristics and outcomes of YAGPC by operator grade are found in Table 1.

\section{Visual acuity}

We found significantly improved VA following YAGPC across the whole cohort as shown in Fig. $1 \mathrm{~A}(p<0.001)$. Figure $2 \mathrm{~A}$ is a bubble plot of pre and post-operative VA. Of the 6308 eyes, $68.1 \%$ VA had improved (above the line), $24.7 \%$ remained with the same visual acuity (on the line), and $7.3 \%$ had a reduction of VA (underneath the line). Of the patients with a pre-operative VA $>0.9$ LogMAR, $31.3 \%$ achieved a final VA of $<0.30$ LogMAR units. Figure $2 B$ shows the change in visual outcomes by 0.30 LogMAR units. We found that of the patients with an excellent pre-operative VA $(\leq 0.00)$, $8.1 \%$ experienced a worsening in VA postoperatively. Additionally, $96.1 \%$ of patients who had a pre-operative VA of $\leq 0.30$ LogMAR, had a better or equal VA post YAGPC. In our cohort, 151 (4.3\%) patients experienced visual loss (0.30 LogMAR unit reduction in VA) in the year following their YAGPC. Of these, 77/151 (51.0\%) showed ocular co-morbidities preoperatively while 74/151 (49.0\%) did not. From those that had no prior ocular co-morbidities, 43/74 $(58.1 \%)$, had treatment for a variety of ocular pathologies (diabetic maculopathy and retinopathy, age-related macular degeneration, glaucoma, retinal detachment and macula-hole development in one patient).

Visual outcomes by operator grade are found in Fig. 1. Although a Kruskal Wallis test demonstrates a difference in LogMAR gain across operator grade, a pairwise analysis with Bonferroni correction does not demonstrate any significant difference across groups.

To further explore these differences in visual outcomes, a multivariate linear regression model is reported in Table 2 . This revealed no difference in VA gain between the ANP and the other operator grades except for the residents' group who showed a significantly higher VA improvement than the ANP $(p=0.020)$. Ocular co-morbidities and increased age were both strongly correlated to a reduced VA gain $(p<0.001$ in both instances, Table 2A)

To explore why residents had a higher VA improvement than the other groups, we repeated the linear regression analysis after excluding patients with ocular co-morbidities and we found no difference between any of the groups.

\section{Risk factors for requiring further YAGPC}

We found $95(1.5 \%)$ required repeat YAGPC. A summary of risk factors leading to repeat YAGPC can be found in Table 3. Younger patients, ocular co-morbidities and ophthalmology operator were associated with higher proportion of those requiring further YAGPC. After dichotomising operator grade to ophthalmologists and ANP, we found that ANP had significantly lower proportion of patients requiring further YAGPC compared to ophthalmologists $(p<0.001)$.

As our ophthalmology subgroup had a wide range of experience, with variety in patients encountered between groups, such as age and ocular co-morbidities, we performed a multivariate binary logistic regression analysis with repeat YAGPC as the dependent variable and this is included in Table 2B. SAS ( $p=$ $0.029)$, residents $(p=0027)$, fellows $(p=0.041)$, and consultants $(p$ $=0.012$ ) all had significantly higher repeat YAGPC rate compared to the ANP. Finally, we found a reduced YAGPC rate with increasing age $(p<0.001)$

\section{Complications}

We reported complications across all our cohort in Table 1. Most of our patients $(99.0 \%)$ had no complications. We had $13(0.2 \%)$ cases of cystoid macular oedema (CMO), a temporary rise in intraocular pressure in $26(0.4 \%)$ patients, $22(0.3 \%)$ developed uveitis and 4 $(0.1 \%)$ cases suffered a detached retina. No significance was found 
Table 1. Operator Grade and YAG PC baseline characteristics and outcomes.

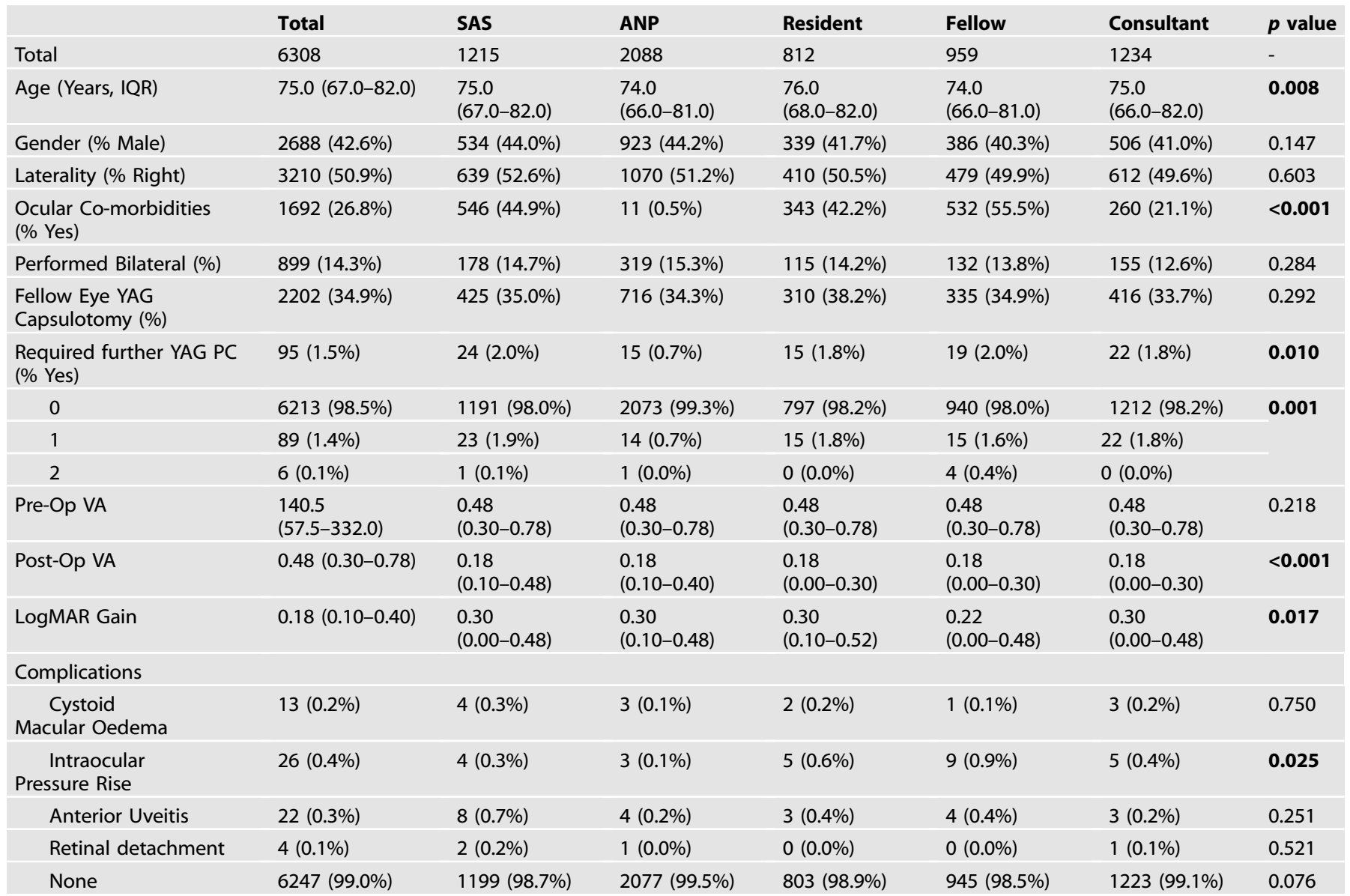

Age and VA are reported as median (interquartile range) and Kruskal-Wallis test used to compare continuous variables. Chi-Squared analysis is used for comparing more than two nominal groups. Statistical significance in bold.
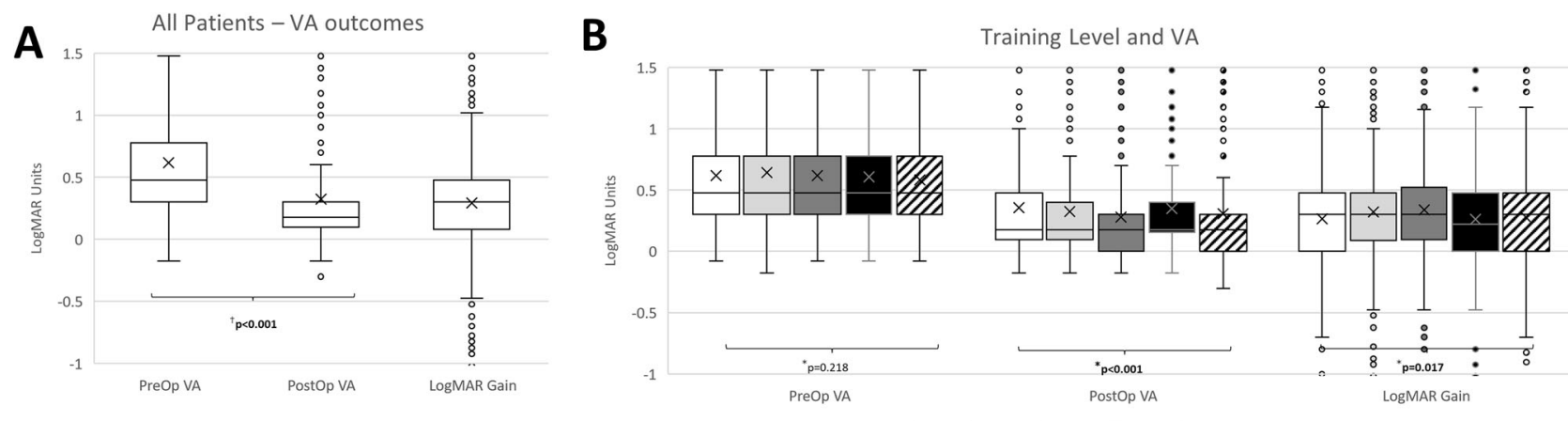

$\square$ SAS $\square$ ANP $\square$ Trainee $\mathbf{\square}$ Fellow $\square$ consultant

Fig. 1 Box and whisker plot of visual acuity outcomes. Box and Whisker plot. ' $X$ ' denotes mean. *Independent-Samples Kruskal-Wallis test, ${ }^{+}$Wilcoxon-Signed Rank Test. Statistical significance in bold.

in complication rate between different training levels $(p=0.076)$. All complications were temporary and managed with medical management other than the retinal detachment that required surgical intervention.

\section{DISCUSSION}

Our study provides pragmatic 'real-world' outcomes of 6308 eyes that underwent a primary YAG PC in a tertiary referral centre. We assessed the outcomes of ANP compared to ophthalmologists in performing YAGPC by looking at visual outcomes, repeat YAGPC and complication rate following YAGPC. To the best of our knowledge, this study represents the first series to assess the outcomes of ANP compared to ophthalmologists in YAGPC and it is the largest series to date assessing visual outcomes in YAGPC.

Allied health professionals have had an integral role across many disciplines of medicine [25]. They have been successfully integrated into emergency eye services to help during the COVID-19 pandemic [26] and the role of their integration in ophthalmic care has been discussed with in the US healthcare system [27]. The first ANP in the 


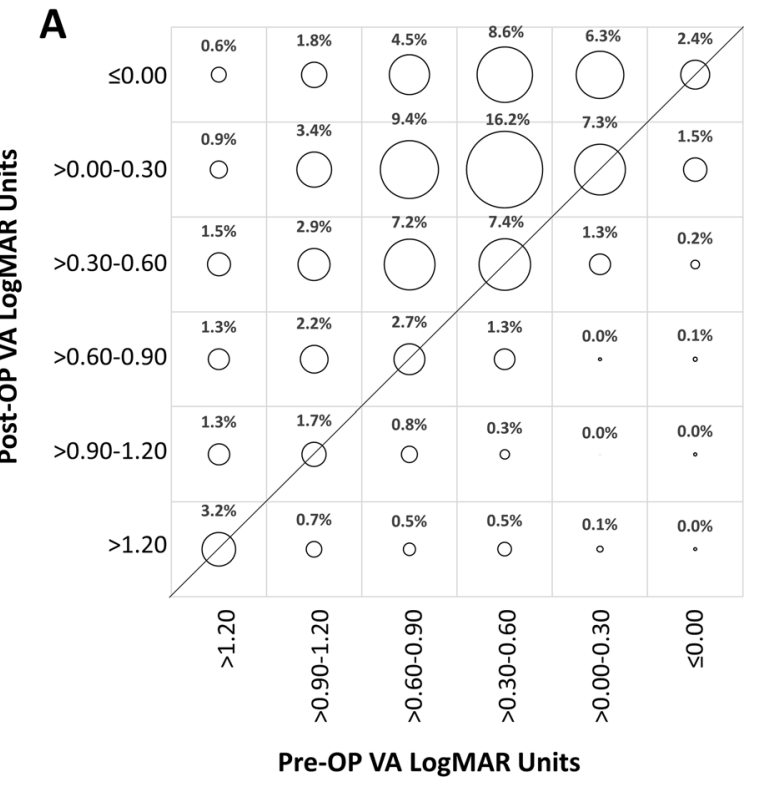

B

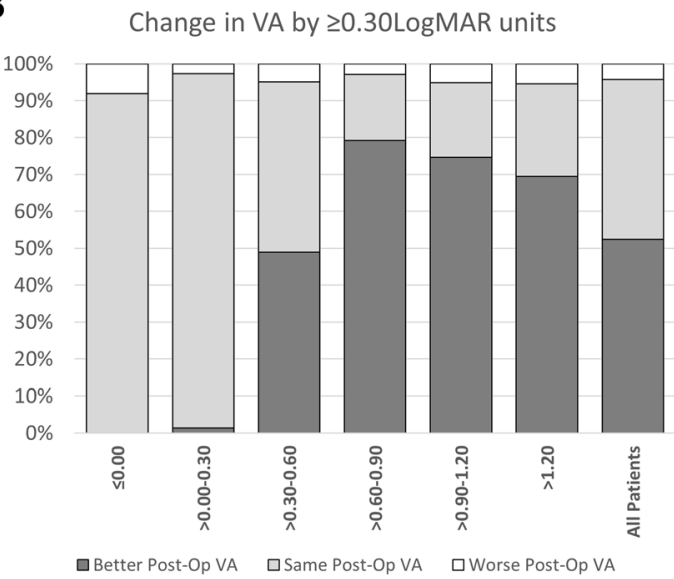

(A) Bubble plot of visual acuities for all eyes. The size of each circle is proportional to the total number of observations and the labels are the percentage of eyes. $68.1 \%$ were in a higher VA category (above the line), $24.7 \%$ remained in the same category (on the line) and $7.3 \%$ were in a lower VA category after YAGPC.

(B) Favourable outcomes are seen across all pre-Op VA subgroups. Better and Worse VA were based on changes of 0.30 or more LogMAR units.

Fig. 2 Comparison of pre and post-YAGPC visual acuity. A Bubble plot of visual acuities for all eyes. The size of each circle is proportional to the total number of observations and the labels are the percentage of eyes. $68.1 \%$ were in a higher VA category (above the line), $24.7 \%$ remained in the same category (on the line) and 7.3\% were in a lower VA category after YAGPC. B Favourable outcomes are seen across all preOp VA subgroups. Better and Worse VA were based on changes of 0.30 or more LogMAR units.

Table 2. (A) Multivariate linear regression model for LogMAR gain post YAGPC. (B) Multivariate binary logistic regression model for repeat YAGPC.

\section{Independent variable}

(A)

Ethnicity (REF Black)

White

SA

$-0.024(-0.059$ to 0.011$)$

$-0.037(-0.085$ to 0.012$)$

Operator Grade (REF ANP)

SAS

Resident

Fellow

Consultant

Ocular Co-morbidity

Age

Independent variable

(B)

Ethnicity (REF White)

$\begin{array}{ll}\text { Black } & 0.406 \\ \text { SA } & 0.035\end{array}$

0.035

0.819

0.885

0.808

0.894

0.425

$-0.042$
-0.017 ( -0.062 to 0.029$)$

0.057 (0.009 to 0.106$)$

-0.018 ( -0.070 to 0.033$)$

-0.034 ( -0.078 to 0.010$)$

$-0.068(-0.104$ to -0.032$)$

$-0.003(-0.004$ to -0.002$)$

B coefficient
Age $p$ value

0.171

0.137

0.469

0.020

0.485

0.128

$<0.001$

$<0.001$

Odds ratio $(95 \% \mathrm{Cl})$

$p$ value

1.501 (0.950 to 2.372$) \quad 0.082$

1.035 (0.517 to 2.075$) \quad 0.922$

$2.268(1.090$ to 4.720$) \quad \mathbf{0 . 0 2 9}$

$2.423(1.103$ to 5.321$) \quad \mathbf{0 . 0 2 7}$

2.243 (1.032 to 4.876$) \quad \mathbf{0 . 0 4 1}$

2.446 (1.217 to 4.916$) \quad \mathbf{0 . 0 1 2}$

1.530 (0.948 to 2.468$) \quad 0.082$

0.959 (0.946 to 0.971$) \quad<0.001$

$S A$ South Asian, REF reference variable.

Significance defined as $p<0.05$. Significant values in bold.

(A) LogMAR gain: No difference between ethnicities and LogMAR gain. No difference between ANP and other operator grades for LogMAR gain in VA other than residents who had significantly higher LogMAR gain than ANP $(p=0.020)$. Ocular co-morbidities and increased Age were both strongly correlated to reduced LogMAR gain $(p<0.001$ in both instances).

(B) Repeat YAGPC: No difference in ethnicity and repeat YAGPC rate. SAS $(p=0.029)$, residents $(p=0027)$, fellows $(p=0.041)$, and consultants $(p=0.012)$ all had significantly higher repeat YAGPC rate compared to ANP. Increased age had reduced YAGPC rate $(p<0.001)$. 
Table 3. Differences in characteristics and outcomes of eyes with primary YAGPC and those requiring further YAGPC.

\begin{tabular}{|c|c|c|c|}
\hline & $\begin{array}{l}\text { No Further } \\
\text { YAGPC } 6213 \\
(98.5 \%)\end{array}$ & $\begin{array}{l}\text { Further YAGPC } \\
95(1.5 \%)\end{array}$ & $p$ value \\
\hline Age (years, IQR) & $\begin{array}{l}75.0(67.0 \\
\text { to } 82.0)\end{array}$ & $\begin{array}{l}66.0(51.0 \\
\text { to } 78.0)\end{array}$ & $<0.001$ \\
\hline \multicolumn{4}{|l|}{ Ocular co-morbidity (\% Yes) } \\
\hline No & 4565 (98.9\%) & $51(1.1 \%)$ & $<0.001$ \\
\hline Yes & 1648 (97.4\%) & $44(2.6 \%)$ & \\
\hline \multicolumn{4}{|l|}{ Gender } \\
\hline Male & 2649 (98.5\%) & $39(1.5 \%)$ & 0.835 \\
\hline Female & 3564 (98.5\%) & $56(1.5 \%)$ & \\
\hline \multicolumn{4}{|l|}{ Operator Grade } \\
\hline ANP & 2073 (99.3\%) & $15(0.7 \%)$ & $<0.001$ \\
\hline Ophthalmologist & 4140 (98.1\%) & $80(1.9 \%)$ & \\
\hline SAS & 1191 (98.0\%) & $24(2.0 \%)$ & 0.982 \\
\hline Resident & 797 (98.2\%) & $15(1.8 \%)$ & \\
\hline Fellow & $940(98.0 \%)$ & $19(2.0 \%)$ & \\
\hline Consultant & $1212(98.2 \%)$ & $22(1.8 \%)$ & \\
\hline Pre-YAGPC VA (LogMAR) & $\begin{array}{l}0.48(0.30 \\
\text { to } 0.78)\end{array}$ & $\begin{array}{l}0.48(0.30 \\
\text { to } 1.00)\end{array}$ & 0.188 \\
\hline Post-YAGPC VA (LogMAR) & $\begin{array}{l}0.18(0.10 \\
\text { to } 0.30)\end{array}$ & $\begin{array}{l}0.30(0.18 \\
\text { to } 0.78)\end{array}$ & $<0.001$ \\
\hline LogMAR Gain & $\begin{array}{l}0.30(0.08 \\
\text { to } 0.48)\end{array}$ & $\begin{array}{l}0.18(0.00 \\
\text { to } 0.48)\end{array}$ & 0.023 \\
\hline
\end{tabular}

ANP advanced nurse practitioner.

Data are reported as median (interquartile range). Mann-Whitney $U$ test was used to compare Age between groups. Chi-Squared ( $>2$ groups) and fisher-exact test (2 groups) were otherwise used to compare nominal groups. Statistical significance in bold.

UK performed YAGPC in 1997 at the Hinchingbrooke Hospital in Cambridgeshire following a training programme [28]. We included the training requirements and journey that our ANP underwent to be a competent YAG capsulotomy operator. While we detected no difference in visual outcomes between different operator grades, we found that a lower proportion of patients in the ANP group required further YAGPC compared to the ophthalmologists' group $(p<0.001$ in both instances). After including ethnicity, age and ocular comorbidities in a multivariate binary logistic analysis, the ANP still had a significantly lower rate of repeat YAGPC. $33.1 \%$ of our primary YAGPCs were performed by a single ANP. We hypothesize that the improved outcomes are linked to the higher volume of cases and therefore greater experienced of the ANP.

Mohamed et al. [22] compared patient satisfaction of nurse-led vs consultant-led intravitreal injection treatment (IVT) and found equivalent results in both groups. They suggested an update of the Royal College of Ophthalmologists guidelines to reflect the contribution of ANPs in delivering a high level of care. Raman et al. explored the safety profile of nurses injecting Ozurdex implants with similar excellent outcomes and high patients' satisfaction [29]. However, no study has assessed the outcomes of nurses performing YAGPC yet.

We found a male minority of $42.6 \%$ which is in line with less males having had cataract surgery (males $42.0 \%$ across 240,803 surgeries in 2019) as found by the NOD Audit, UK [30]. Our median visual improvement of 0.30 (IQR 0.08-0.48) LogMAR units is consistent with other case series [31-35]. The largest case series assessing visual outcomes following YAGPC by Stark et al. (1985) in a study involving 2110 patients found that the postoperative VA was $20 / 40$ (LogMAR 0.30 ) or better in $81 \%$ patients while $84 \%$ experienced an improvement in their VA [34]. Bath et al. (1986) after assessing 3711 patients demonstrated that $92.2 \%$ of them had an VA gain following YAGPC. However, they excluded patients with several ocular co-morbidities.
When assessing visual outcomes following cataract surgery, according to the NOD [30], the median pre-operative VA, the postoperative VA and the gain in VA were 0.50 (range; $-0.30-\mathrm{NPL}$ ), 0.10 (range; $-0.30-N P L)$ and 0.38 LogMAR units (IQR; $0.20-0.60$ gain), respectively. These are comparable to our findings of median preoperative, post-operative, and gain in VA of 0.48 (IQR; $0.30-0.78$ ), 0.18 (IQR; 0.10-0.40) and 0.30 (0.08-0.48) LogMAR units, respectively. The comparison between VA outcomes in cataract surgery nationally from the NOD data and our study suggests that YAGPC has a significant impact on vision which is comparable to that provided by cataract surgery.

Over the past 20 years there has been a dramatic improvement in the safety of cataract extraction surgery and this operation is offered to patients with increasingly better preoperative VAs. This can be attributed to several factors including better surgical techniques and outcomes, patients' expectations and their need for higher visual function to maintain independence [36]. We have shown in Fig. 2B, that a pre-operative VA of $\leq 0.00$ LogMAR units led to a lower percentage of patients with a notable improvement in their vision. This is also echoed by near identical bubble plots of pre and post-operative VA in our data. Similar outcomes also apply to the VA data on cataract surgery published by the NOD [23]. However, these results do not account for contrast sensitivity, an important metric not commonly measured in clinical practice [33].

Our complication rate is similar to that published in the literature [21, 37-40] and we report an excellent safety profile across our cohort. We did not find a difference in over-all complication rate and training level.

\section{Study limitation and strengths}

Our study is retrospective in nature and so we had no standardised treatment protocol to follow. Therefore, the proportion of patients receiving post-operative topical drops, and total energy power was not retrievable from our database. Nevertheless, post-operative steroid eye drops are not routinely recommended following YAG posterior capsulotomy (YAGPC) in our unit or other published literature, and they are only recommended in cases with high energy use and relevant ocular co-morbidities [40]. Additionally, we only reported on the outcome of a single ANP. However, this paper describes the steps required by our ANP to reach this level of competence and the extraordinary volume of YAGPCs that an ANP can perform.

Our study also has several strengths. A retrospective analysis allowed us to collate the largest recent case series the authors are aware of assessing VA outcomes following YAGPC. In addition, this is the first paper assessing the outcomes of ANPs performing YAGPC.

\section{CONCLUSION}

Our study demonstrates that ANPs can deliver safe and effective YAGPC. We also showed that YAGPC leads to excellent visual outcomes. The VA improvement is comparable to the UK national data following cataract surgery. We are hopeful that this study will facilitate the integration of ANP-lead YAG services across other eye units.

\section{Summary}

What was known before

- Advanced Nurse Practitioners (ANP) can safely and effectively contribute to various aspects of ophthalmic healthcare (i.e. intravitreal injections)

- YAG posterior capsulotomy (YAGPC) is a common sequelae of cataract surgery

- YAGPC can provide good visual improvements 
What this study adds

- This is the largest series to date assessing visual outcomes after YAGPC and shows excellent results.

- ANPs can safely contribute to a YAGPC service, providing a low repeat YAGPC rate relative to ophthalmologists

\section{REFERENCES}

1. Findl $O$, Buehl W, Bauer $P$, Sycha T. Interventions for preventing posterior capsule opacification. Cochrane Database Syst Rev. 2010;2017:CD003738.

2. Raj SM, Vasavada AR, Kaid JSR, Vasavada VA, Vasavada VA. Post-operative capsular opacification. Nepal J Ophthalmol: Biannu Peer-reviewed Acad J Nepal Ophthal Soc: NEPJOPH. 2009;1:43-59.

3. Trivedi RH, Werner L, Apple DJ, Pandey SK, lzak AM. Post cataract-intraocular lens (IOL) surgery opacification. Eye. 2002;16:217-241.

4. Ling R, Borkenstein EM, Borkenstein AF. Evaluation of nd:Yag laser capsulotomy rates in a real-life population. Clin Ophthalmol. 2020;14:3249-57.

5. Nishi O, Nishi K, Wickström K. Preventing lens epithelial cell migration using intraocular lenses with sharp rectangular edges. J Cataract Refractive Surg. 2000;26:1543-49.

6. Nishi O, Nishi K, Akura J, Nagata T. Effect of round-edged acrylic intraocular lenses on preventing posterior capsule opacification. J Cataract Refractive Surg. 2001;27:608-13.

7. Peng Q, Visessook N, Apple DJ, Pandey SK, Werner L, Escobar-Gomez M, et al. Surgical prevention of posterior capsule opacification: Part 3: Intraocular lens optic barrier effect as a second line of defense. J Cataract Refractive Surg. 2000;26:198-213.

8. Kohnen T, Fabian E, Gerl R, Hunold W, Hütz W, Strobel J, et al. Optic Edge Design as Long-term Factor for Posterior Capsular Opacification Rates. Ophthalmology. 2008;115:1308-14.e3.

9. Buehl W, Findl O. Effect of intraocular lens design on posterior capsule opacification. J Cataract Refractive Surg. 2008;34:1976-85.

10. Buehl W, Findl O, Menapace R, Rainer G, Sacu S, Kiss B, et al. Effect of an acrylic intraocular lens with a sharp posterior optic edge on posterior capsule opacification. J Cataract Refractive Surg. 2002;28:1105-11.

11. Buehl W, Findl O, Menapace R, Sacu S, Kriechbaum K, Koeppl C, et al. Long-term effect of optic edge design in an acrylic intraocular lens on posterior capsule opacification. J Cataract Refractive Surg. 2005;31:954-61.

12. Buehl W, Menapace R, Findl O, Neumayer T, Bolz M, Prinz A. Long-term Effect of Optic Edge Design in a Silicone Intraocular Lens on Posterior Capsule Opacification. Am J Ophthalmol. 2007;143:913-9.e2.

13. Nishi O, Yamamoto N, Nishi K, Nishi Y. Contact inhibition of migrating lens epithelial cells at the capsular bend created by a sharp-edged intraocular lens after cataract surgery. J Cataract Refractive Surg. 2007;33:1065-70.

14. Auffarth GU, Golescu A, Becker KA, Völcker HE. Quantification of posterior capsule opacification with round and sharp edge intraocular lenses. Ophthalmology. 2003;110:772-80.

15. Findl O, Buehl W, Menapace R, Sacu S, Georgopoulos M, Rainer G. Long-term effect of sharp optic edges of a polymethyl methacrylate intraocular lens on posterior capsule opacification: A randomized trial. Ophthalmology.2005;112:2004-8.

16. Mester U, Fabian E, Gerl R, Hunold W, Hütz W, Strobel J, et al. Posterior capsule opacification after implantation of CeeOn Edge 911A, PhacoFlex SI-40NB, and AcrySof MA60BM lenses: One-year results of an intraindividual comparison multicenter study. J Cataract Refractive Surg. 2004;30:978-85.

17. Tetz M, Wildeck A. Evaluating and defining the sharpness of intraocular lenses: Part 1: Influence of optic design on the growth of the lens epithelial cells in vitro. J Cataract Refractive Surg. 2005;31:2172-9.

18. BuehI W, Menapace R, Sacu S, Kriechbaum K, Koeppl C, Wirtitsch M, et al. Effect of a silicone intraocular lens with a sharp posterior optic edge on posterior capsule opacification. J Cataract Refractive Surg. 2004;30:1661-7.

19. Ursell PG, Dhariwal M, O'Boyle D, Khan J, Venerus A. 5 year incidence of YAG capsulotomy and PCO after cataract surgery with single-piece monofocal intraocular lenses: a real-world evidence study of 20,763 eyes. Eye (Basingstoke). 2020;34:960-8.

20. Wesolosky JD, Tennant M, Rudnisky CJ. Rate of retinal tear and detachment after neodymium:YAG capsulotomy. J Cataract Refractive Surg. 2017;43:923-8.

21. Elbaz U, Hakkala L, Hecht I, Achiron A, Gershoni A, Tuuminen R. Nd:YAG capsulotomy is not a risk factor for retinal detachment after phacoemulsification cataract surgery. Acta Ophthalmologica. 2021;99:aos.14757.
22. Mohamed R, Ramcharan D, Srikaran S, Mensch E. A model of clinical practice: a randomised clinical study evaluating patient satisfaction of nurse-led vs consultant-led intravitreal injection. Eye (Basingstoke). 2018;32:1148-9.

23. Day AC, Donachie PHJ, Sparrow JM, Johnston RL. The Royal College of Ophthalmologists' National Ophthalmology Database study of cataract surgery: Report 1, visual outcomes and complications. Eye (Basingstoke). 2015;29:552-60.

24. Moussa G, Bassilious K, Mathews N. A novel excel sheet conversion tool from Snellen fraction to LogMAR including 'counting fingers', 'hand movement', 'light perception' and 'no light perception' and focused review of literature of low visual acuity reference values. Acta Ophthalmologica. 2021;99:aos.14659.

25. McCrory G, Patton D, Moore Z, O'Connor T, Nugent L. The impact of advanced nurse practitioners on patient outcomes in chronic kidney disease: a systematic review. J Ren Care. 2018;44:197-209.

26. Moussa G, Mushtaq F, Mandal P, Mathews N, Royal B, Manjunatha N, et al. Restructuring emergency eye services during COVID-19 in a tertiary referral centre. Br J Hospital Med. 2020;81:1-8.

27. Browning DJ. Physician Assistants and nurse practitioners in ophthalmologyhas the time come? Am J Ophthalmol. 2018;186:ix-xi.

28. Gibbons H. Nurses performing YAG laser capsulotomy - the UK perspective. Int J Ophthalmic Pract. 2010;1:4-4.

29. Raman V, Triggol A, Cudrnak T, Konstantinos P. Safety of nurse-led intravitreal injection of dexamethasone (Ozurdex) implant service. Audit of first 1000 cases. Eye. 2021;35:388-92.

30. RCOphth. Year 5 Annual Report - The Fourth Prospective Report of the National Ophthalmology Database Audit NHS or equivalent Funded Cataract Surgery: 01 September 2018 to 31 August 2019.; 2020.

31. Sirisha G, Lakshmi Chowdary N. Visual outcome of nd-yag laser capsulotomy in posterior capsule opacification. J Evolution Med Dent Sci. 2016;5:1479-82.

32. Hossain M, Hossain M, Hossain M. Visual Outcome after Nd:YAG Laser Capsulotomy. J Armed Forces Med Coll Bangladesh. 1970;5:29-31.

33. Hayashi K, Hayashi H, Nakao F, Hayashi F. Correlation between posterior capsule opacification and visual function before and after Neodymium:YAG laser posterior capsulotomy. Am J Ophthalmol. 2003;136:720-6.

34. Stark WJ, Worthen D, Holladay JT, Murray G. Neodymium:YAG Lasers: An FDA Report. Ophthalmology.1985;92:209-12.

35. Ghasemi Falavarjani K, Aghamirsalim M, Abri Aghdam K. Resident-performed neodymium: YAG laser posterior capsulotomy. Can J Ophthalmol. 2017;52:324-30.

36. Desai P. Cataract surgery: One or both eyes? Br J Ophthalmol. 2012;96:1279-80.

37. Kolli H, Evers C, Murray PI. Nd:YAG Laser Posterior Capsulotomy in Adult Patients with Uveitis. Ocular Immunology and Inflammation. 2021;29:1537-9.

38. Shetty NK. Study of Variation in Intraocular Pressure Spike (IOP) Following NdYAG Laser Capsulotomy. J Clin Diagn Res. 2016;12:NC09-NC12.

39. Ari S, Cingü AK, Sahin A, Çinar Y, Çaça I. The Effects of Nd:YAG Laser posterior capsulotomy on macular thickness, intraocular pressure, and visual acuity. Ophthalmic Surgery Lasers, Imaging. 2012;43:395-400.

40. Snyder LA, Chou TY. The Incidence of Iritis and the use of topical antiinflammatory drops after Nd:YAG Posterior Capsulotomy. Investigative Ophthalmol Vis Sci. 2002;43:374.

\section{AUTHOR CONTRIBUTIONS}

All authors have made substantial contributions to all the following: (1) the conception and design of the study, or acquisition of data, or analysis and interpretation of data, (2) drafting the article or revising it critically for important intellectual content, (3) final approval of the version to be submitted.

\section{COMPETING INTERESTS}

The authors declare no competing interests.

\section{ADDITIONAL INFORMATION}

Correspondence and requests for materials should be addressed to George Moussa.

Reprints and permission information is available at http://www.nature.com/ reprints

Publisher's note Springer Nature remains neutral with regard to jurisdictional claims in published maps and institutional affiliations. 